\title{
In the porcine placenta, aromatase (CYP19) is specifically expressed in trough-like structures of the trophoblast at the basis and flanks of chorionic folds*
}

\author{
PERSHOTAM KHATRI ${ }^{1}$, GERHARD SCHULER ${ }^{2}$, \\ ELÇIN ÖZOCAK-BATMAZ ${ }^{3}$, GÖZDE R. ÖZALP ${ }^{4}$
}

\author{
'Department of Animal Reproduction, Faculty of Animal Husbandry and Veterinary Sciences (FAHVS), \\ Sindh Agriculture University, Tandojam, Sindh 70060, Pakistan \\ ${ }^{2}$ Clinic for Obstetrics, Gynecology and Andrology of Large and Small Animals, \\ Faculty of Veterinary Medicine, Justus-Liebig-University, Giessen, Germany \\ ${ }^{3}$ Department of Surgery, Faculty of Veterinary Medicine, ${ }^{4}$ Department of Obstetrics and Gynecology, \\ Faculty of Veterinary Medicine, Uludag University, Bursa, Turkey
}

Pershotam K., Schuler G., Özocak-Batmaz E., Özalp G. R.

In the porcine placenta, aromatase (CYP19) is specifically expressed in trough-like structures of the trophoblast at the basis and flanks of chorionic folds

Summary

Aromatase was localized in the porcine placenta by immunohistochemistry during the first peak of maternal estrogen concentrations (D25; $n=3)$, their nadir around midgestation $(D 50 ; n=3)$ and their second increase in late gestation (D110; $n=3)$. On D25, aromatase was highly expressed throughout the trophoblast. On D50, its expression was restricted to the trophoblast lining the trough-like fossal regions between the bases of chorionic folds. However, immunostaining was detected only in a minor proportion of these locations, with staining intensity being generally only weak to moderate. On D110, distinct to intense immunostaining was found in virtually all trophoblast fossal regions and in similar structures at the flanks of chorionic folds adjacent to the free margins of secondary and tertiary endometrial folds. A weak staining of questionable specificity was occasionally observed in endometrial glands. In other cell types of the utero-placental compartment, aromatase was undetectable. The results suggest that in pigs placental estrogens could be involved in the formation of a more complex geometry of the feto-maternal interface as pregnancy progresses.

Keywords: pig, placenta, trophoblast, aromatase, estrogen

Basically, the porcine placenta is classified as a diffuse epitheliochorial type (17). However, the process of placentation, the morphology of the developed porcine placenta and its dynamic morphological changes throughout gestation are clearly more complex than reflected by this expression. Thus, only basic features will be pointed out here, and for specific information the reader is referred to more detailed descriptions (e.g. in ref. no. 8, 10, 11 and Hopkins K. L.: In vitro characterization of aromatase, estrone sulfotransferase and estrone sulfatase activities in the porcine placenta and endometrium at 30 ,

* With support from the German Academic Exchange Service (DAAD) re-invitation program for former scholarship holders to PK.
60 and 90 days of gestation. Master thesis, Virginia Polytechnic Institute and State University, Blacksburg, Virginia, USA. http://vtechworks.lib.vt.edu/bitstream/ handle/10919/45793/LD5655.V855 1987.H664.pdf? sequence $=1 \&$ isAllowed $=y$ ) and the literature cited herein. The chorion of the developed porcine placenta can be divided into the placental zone, the laterally adjacent paraplacental zones and the avascular extremities of the gestational sacs. The large placental zone, where the intimate feto-maternal interdigitation takes place, occupies the central region of the sac and contains numerous chorionic folds interlocked with corresponding endometrial folds (rugae). However, above the endometrial gland openings located between 
the folds, the trophoblast is not in close contact with the endometrium, but forms specialized structures, named areolae, for the absorption of histiotrophe $(10,11)$.

In many ungulate species, gestation is associated with the production of significant amounts of pregnancy-associated estrogens. With the exception of the immediate prepartal period, in which they have been related to the preparation of the myometrium and birth canal for parturition, virtually no definite information is available on their biological role throughout the long preceding phase of pregnancy. Observations from various species point to significantly different situations concerning the spectrum of estrogens formed, their profiles in maternal blood and the underlying synthetic pathway (22-24).

In some species, including the pig, considerable amounts of estrogens are already produced by blastocysts or perimplantation conceptuses $(3,4)$. During early porcine gestation, pregnancy-associated estrogens have been suggested to be involved in the control of various important processes. For decades, it was considered certain that estrogens produced by blastocysts around gestational day 11-12 represent the primary antiluteolytic signal that ensures maintenance of luteal function in the sense of a signal for maternal recognition of pregnancy (2). However, the underlying mechanisms are still poorly understood on a molecular level $(16,26,27)$. Moreover, there is evidence of a role in placental expansion (15), fetal water and electrolyte movement (13) and uterine blood flow (9). Again, the exact molecular mechanisms underlying these effects still await further elucidation. Recently, however, the above concepts have been challenged, in some cases substantially, by observations in pregnancies with CYP19A $1^{-/-}$conceptuses. These observations suggest that conceptus estrogens are required for maintenance of pregnancy beyond day 30, but are not essential for preimplantation development, conceptus elongation, and early maintenance of the corpus luteum $(12,18)$.

Despite the important role of pig breeding in meat production, detailed information on the formation of pregnancy-associated estrogens after completion of implantation around day (D) 25 is still sparse for this species. In maternal blood, estrogen concentrations show a pronounced biphasic pattern. The first increase becomes detectable in maternal blood during the third week of gestation, followed by peak concentrations between D23 and D30. After a nadir around D50, estrogen concentrations increase again, reaching maximum concentrations at parturition. Whereas during the first peak virtually only sulfonated forms of estrogens are detected, after D50 free and sulfonated estrogens increase at similar concentrations (19-21).

Activities of enzymes involved in the production of pregnancy-associated estrogens have been characterized in porcine placental tissue in vitro (for comprehensive review, see ref. no. 14). However, to date only very little information is available on their expression patterns on a cellular level, and most of it is related to the blastocyst. Conley et al. (4) investigated the expression of CYP17A1 and aromatase in porcine blastocysts around D12 post mating and found specific changes in the spatial expression patterns of the trophoblast concomitant with development. In addition to the trophoblast, aromatase was also localized in the hypoblast. To our knowledge, virtually no information is available on the temporal and spatial expression patterns of steroidogenic key enzymes in the porcine placenta and endometrium after the completion of placentation. Only one report was found suggesting a steroidogenic activity of the high columnar trophoblast in fossal regions of the porcine placenta, which are the trough-like basal parts of the chorioallantoic folds in the placental zone. Here, ultrastructural features characteristic of steroidogenic cells, such as lipid droplets and whorls of strongly convoluted smooth endoplasmatic reticulum, were observed, and a considerable $3 \beta$-hydroxysteroid dehydrogenase activity was found by histochemistry (7). However, detailed knowledge of the expression pattern of steroidogenic enzymes on a cellular level must be considered as a prerequisite to understand the synthesis and possible roles of pregnancy-associated estrogens. Thus, the aim of this study was to characterize the expression pattern of aromatase, which is the key enzyme in estrogen synthesis, in the porcine uteroplacental compartment. In order to assess conditions around the first estrogen peak in peripheral maternal blood, the midgestational nadir and the immediate prepartal period, the investigations focused on D25, D50 and D110.

\section{Material and methods}

Animals and sample collection. Nine healthy Landrace cross-bred sows aged 3-4 years were included in the study and housed in the farm of the Faculty of Veterinary Medicine in Bursa. Approval for the animal experiments was obtained from the ethics committee of Uludag University (Nr: 201512/08). The sows were observed daily for estrus symptoms and were mated with Landrace cross-bred boars on the first day estrus was detected, designated as D0 of pregnancy. Pregnancy testing was performed by sonographic examination (5-7.5 MHz linear array transducer; Dynamic Imaging MCV-Concept, UK) on D25 after mating. Sows confirmed pregnant were randomly assigned into three groups, each consisting of three animals: D25, D50 and D110. Sample collection was performed under general anesthesia. Sows were starved $12 \mathrm{~h}$ prior to surgery. First, xylazine $\mathrm{HCl}$ (Alfa$\mathrm{zyn}^{\circledR} \% 2$, Alfasan/Ege Vet, Turkey) was administered intramuscularly for sedation $(4 \mathrm{mg} / \mathrm{kg})$. Induction of anesthesia was achieved by the intravenous application of Propofol Lipura 1\% (8 mg/kg, Braun, Melsungen AG, Germany). Then, the animals were intubated with a $9 \mathrm{~mm}$ tube, and anesthesia was maintained with desfluran (Suprane, Baxter, Eczacibasi, Turkey) in oxygen delivered by a vaporizer (AMS 200, Anaesthezia Workstation, Turkey) through an 
adult circle system (AMS 200, Anaesthezia Workstation, Turkey). End-tidal desfluran, carbondioxide, body temperature, pulse oximetry, pulse rate (PR), and ECG were monitored continuously. Intraoperatively, carprofen $(4 \mathrm{mg} / \mathrm{kg}$, Rimadyl ${ }^{\circledR}$, Pfizer, Brazil) was administered via catheter for pain management. The sows were positioned in dorsal recumbency, and a $20-25 \mathrm{~cm}$ incision was made parallel to the udder. The left uterine horn was exposed from the abdominal cavity, and in its midregion a tissue sample was excised consisting of the uterine wall with adherent fetal tissue. The uterine wall, peritoneum, and abdominal musculature were closed with absorbable suture material (Vicryl, Ethicon, 80 Novartis, Switzerland), and the skin with nonabsorbable suture material (Ruschmed, Ipek Plastik, Istanbul, Turkey). Administration of carprofen ( $4 \mathrm{mg} / \mathrm{kg}$, Rimadyl ${ }^{\circledR}$, Pfizer, Brazil) was continued for 3 days postoperatively.

Tissue samples were fixed overnight in $10 \%$ neutral phosphate-buffered formalin. After 20-24 hours of fixation, the tissue samples were transferred to $0.1 \mathrm{M}$ phosphate
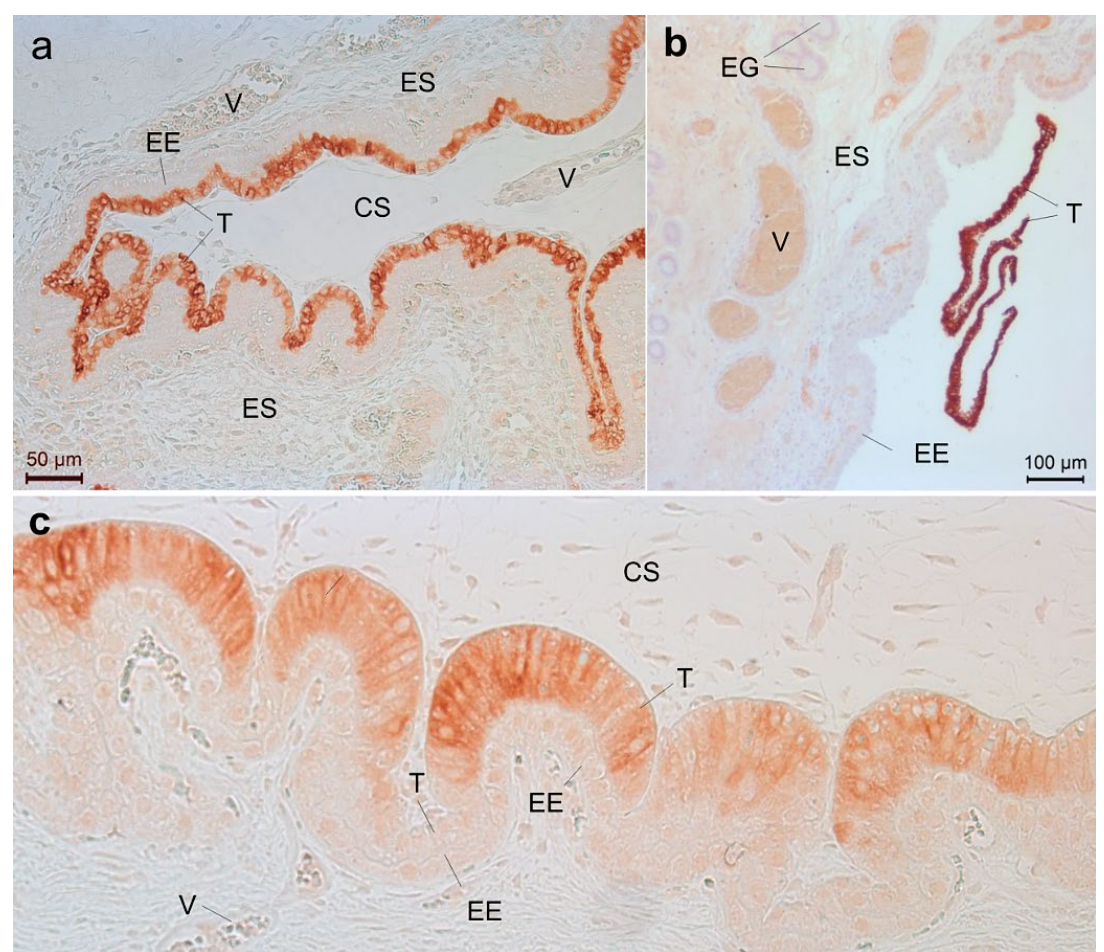

ES

EG

Fig. 1. Immunolocalization of aromatase in utero-placental tissue of pregnant pigs on D25 and D50

Explanations: a) D25; distinct immunostaining in all trophoblast cells (primary antibody: clone 96064); b) D25; intense immunostaining in the trophoblast, which detached itself from the endometrium during the preparation of the tissue sample (primary antibody: H4); c) D50; moderate immunostaining in the highly prismatic trophoblast cells lining the through-like fossae between the bases of chorionic folds. Additionally, weak staining is present in endometrial glands (primary antibody: clone 96064). CS - chorionic stroma, EE - endometrial epithelium, EG - endometrial glands, ES - endometrial stroma, V-blood vessel
$50 \mu \mathrm{m}$

buffer for removal of the fixative and stored in this solution for 1-2 weeks at $4^{\circ} \mathrm{C}$. During this time, the buffer was the samples were embedded in paraffin.

Immunohistochemical procedures. For antigen unmasking, $5 \mu \mathrm{m}$ tissue sections were placed in jars filled with $10 \mathrm{mM}$ citrate buffer $\mathrm{pH} 6.0$, which were heated in vapor phase over boiling water. The temperature in the jars was controlled by a thermometer placed in a reference Jar indirect immunoperoxidase staining method was employed, using the streptavidin-biotin technique for signal enhancement following standard procedures. Immunostaining was performed with two different primary murine monoclonal antibodies, clone H4 (Acris Antibodies, Herford, Germany; Turner et al. 2002; dilution: $1: 500$ ) and clone 96064 (biorbyt, San Francisco, USA; dilution 1:100), to corroborate the specificity of signals obtained. Blocking serum, biotinylated secondary antibody and avidin biotin complex were taken from the Vectastain Elite ABC kit 6102, and the slides were developed with the NovaRED Substrate Kit as specified by the supplier (Vector Laboratories, Burlingame, CA, USA). Phosphate buffered saline $/ 0.3 \%$ Triton X $100 \mathrm{pH}$ 7.4 was used as buffer for dilution of reagents and for washing the slides. In negative controls, buffer was used instead of the primary monoclonal murine antibody. From each animal, 2-3 slides were evaluated. Immunostaining was classified as negative $(-)$, weak $(+)$, moderate $(++)$, distinct $(+++)$, or intense $(++++)$.

\section{Results and discussion}

Identical staining patterns were found with the two primary antibodies applied, clone $\mathrm{H} 4$ and clone 96064 . In porcine testis used as a positive control, aromatase was detected exclusively in the cytoplasm of Leydig cells. Negative controls were free of staining except for the lumen of some blood vessels, where occasional weak nonspecific signals appeared (not shown), apparently associated with residual blood plasma. In utero-placental samples from D25 pregnant animals (Fig. 1a, b), fetal tissues were frequently lost during sample processing because of their weak adherence to the endometrium at this early stage of gestation and their fragile consistency. However, homogeneous distinct to intense cytoplasmic staining was found in all trophoblast cells of the remaining chorionic remnants, which had a uniform cuboidal shape. They were the only cell type in samples from D25 pregnant animals show- 
ing a detectable aromatase expression. In samples from D50 pregnant animals, the feto-maternal contact zone was formed by regularly arranged interdigitated chorionic folds and endometrial rugae (Fig. 1c). Specific cytoplasmic staining occurred in the through-like fossae between the bases of chorionic folds, which were lined by specific, highly prismatic trophoblast cells. However, only a minor proportion of chorionic fossae were clearly positive and showed, at most, moderate to distinct staining intensity, whereas in the vast majority of cases immunostaining was only weak or absent. No signals occurred in the cuboidal trophoblast cells between the fossa regions. Occasionally and depending on the individual tissue sample and location within the slide, weak immunostaining was also observed in endometrial glands, but not in the superficial endometrial epithelial cells (Fig. 1c). In D110 pregnant animals, the feto-maternal contact zone was considerably higher compared to D50 pregnant animals, and the geometric arrangement of fetal or maternal tissue components was significantly more complex (Fig. 2a, b). In all tissue samples investigated, a distinct to intense cytoplasmic immunostaining was found in the trophoblast of virtually all chorionic fossae. Moreover, distinct to intense cytoplasmic staining also occurred in the trophoblast of fossa-like structures situated at the flanks of chorionic folds. Here, the aromatase-positive trophoblast cells showed variable cell height. The cuboidal trophoblast cells outside the fossae or fossa-like regions including the areolae (Fig. 2a), were completely negative, and there was no fluent transition in staining intensity between aromatase-positive cell clusters and adjacent aromatase-negative trophoblast cells. As observed in samples from D50 pregnant animals, weak cytoplasmic staining was present in a variable proportion of endometrial glands. In superficial endometrial epithelium, allantoic endoderm, endometrial and chorionic connective tissue, myometrium and the fetal and maternal vascular system, no specific immunostaining was found. At each gestational age under investigation, consistent staining patterns were found in animals of the corresponding observation group.

In mammals, the CYP19 gene encoding aromatase generally exists only as a single copy. In pigs, however, three CYP19 genes have been identified encoding specific aromatase isoforms in different tissues (preimplantation blastocyst, placental and gonadal/ hypothalamic isoform $(5,6))$. The primary monoclonal antibody H4 used in this study is believed to detect all three porcine aromatase isoforms, as the epitope recognized is fully conserved between the porcine aromatase isoforms $(6,25)$. For the second primary antibody used here, no detailed information is provided by the supplier. However, the staining patterns in porcine testicular and utero-placental tissues were completely identical for the two antibodies, which is a compelling evidence for the specificity of signals observed in the porcine placenta.

Maternal estrogen blood levels during pregnancy (19-21) were found to closely reflect aromatase staining patterns observed in the trophoblast, with a uniform distinct to intense signal in all trophoblast cells on D25, concomitant with the first estrogen peak, followed by a mostly absent or weak to moderate staining confined to the chorionic fossae during the nadir of maternal estrogen concentrations around D50. The distinct prepartal increase in maternal estrogen concentrations was found to be related to a significant upregulation of aromatase expression in the high cylindrical trophoblast cells of virtually all chorionic fossae and fossa-like structures in the late gestational

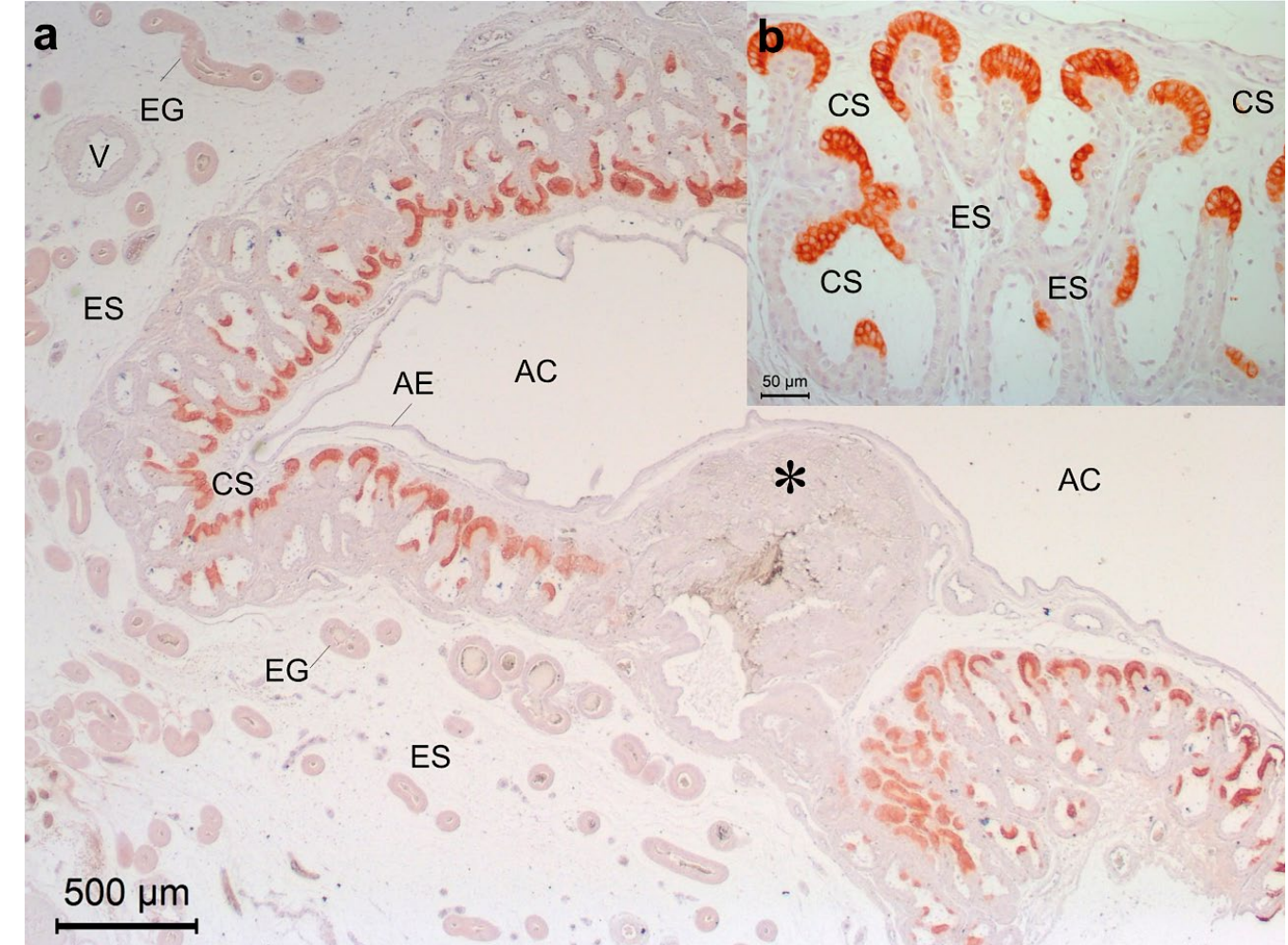

Fig. 2. Immunolocalization of aromatase in utero-placental tissue of pregnant pigs on D110 Explanations: a) Distinct to intense immunostaining is present in practically all fossal regions of chorionic folds, whereas the trophoblast of an areola $(*)$ is completely negative (primary antibody: H4); b) In addition to the fossae between the bases of chorionic folds, distinct to intense immunostaining was also found in fossa-like structures at their flanks (primary antibody: clone 96064). AC - allantoic cavity, AE - amniotic endoderm, CS - chorionic stroma, EG - endometrial glands, ES - endometrial stroma, V - blood vessels 
porcine placenta (Fig. 2, 3). The significant change in aromatase expression pattern, related to the switch from the blastocyst to the placental aromatase isoform and the characteristic estrogen profile measured in maternal blood (19-21), is consistent with the concept that pregnancy-associated estrogens in pigs may have different functions throughout gestation.

In comparison to D50, when chorionic folds and endometrial rugae were arranged in a mostly regular, parallel manner (Fig. 1c, Fig. 3a), on D110 the interdigitation between fetal and maternal tissue was

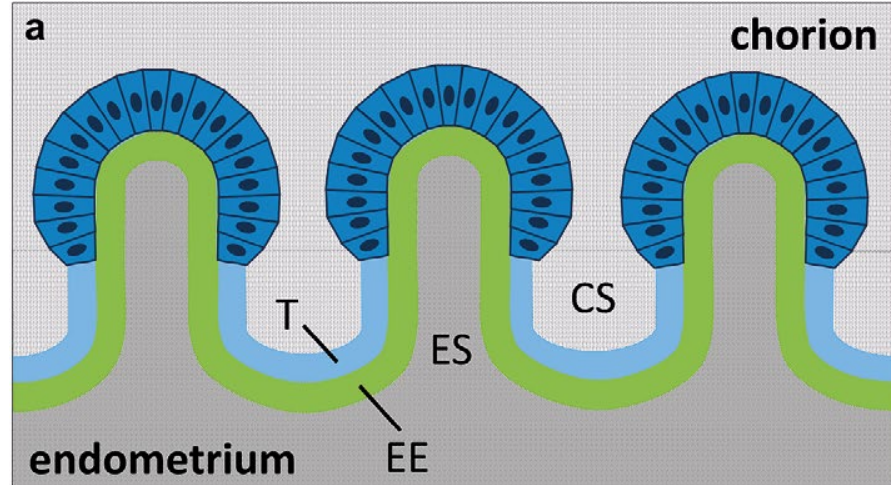

b

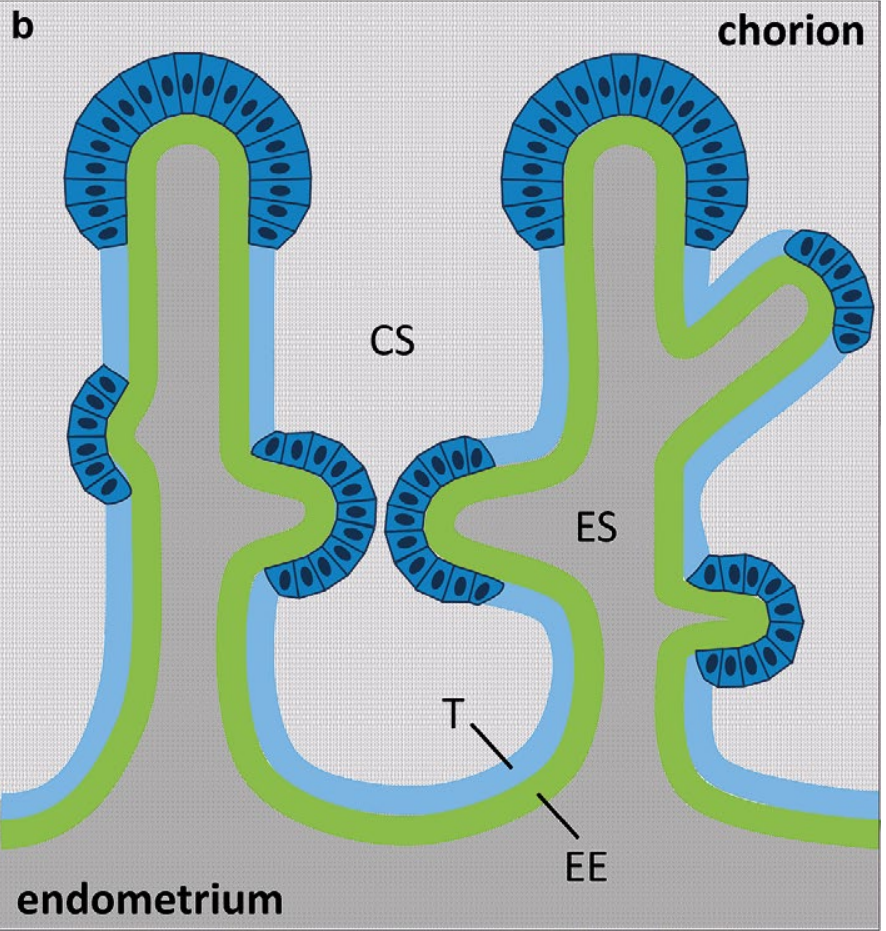

Fig. 3. Schematic representation of the microarchitecture of the feto-maternal contact zone and the location of aromatase-positive trophoblast cells in the porcine placenta (stained dark blue)

Explanations: a) On D50, aromatase is specifically but moderately expressed in the highly prismatic trophoblast cells lining the trough-like fossae between the basal regions of chorionic folds. b) On D110, the chorionic folds and the corresponding endometrial rugae are significantly higher. The formation of secondary and tertiary folds sometimes gives rise to villus-/crypt-like structures. In addition to the fossae between the basal areas of primary chorionic folds, clusters of intensely aromatase-positive trophoblast cells are regularly found at sites adjacent to free margins of secondary and tertiary endometrial folds. CS - chorionic stroma, EE - endometrial epithelium, ES - endometrial stroma, T - trophoblast clearly more intense and complex, with the formation of secondary and tertiary chorionic folds and maternal rugae, partly giving rise to villi-/crypt-like structures. Interestingly, in addition to the trough-like structures between the bases of primary chorionic folds, in late gestational placenta, aromatase was also highly expressed in similar structures located at their flanks, where they were in immediate contact with the free margins of outgrowing secondary or tertiary endometrial rugae (Fig. 2a, b, Fig. 3b). Thus, it is tempting to speculate that in the second half of gestation, after having played their specific roles in maternal recognition of pregnancy and development of the periimplantation conceptuses, placental estrogens may be involved in the differentiation of the feto-maternal contact zone, possibly by controlling the progressive growth and branching of chorionic folds and endometrial rugae. The immunolocalization of aromatase in the trough-like chorionic fossal regions is consistent with earlier observations by electron microscopy and $3 \beta$-hydroxysteroid dehydrogenase histochemistry, which also point to a significant steroidogenic capacity of these specialized trophoblast cells (7).

Like nutritive material from the circulating maternal blood (haemotrophe), secretory products of endometrial glands (histiotrophe) are also an important source of nutrients, which are crucial during the preimplantation period, but may also contribute significantly to the supply of nutrients for the developing fetus until late gestation. In the placenta of various species, such as ruminants, horses and pigs, the trophoblast forms specific structures, named areolae, to absorb secretions from the openings of endometrial glands. Interestingly, as previously described in the equine placenta (1), aromatase expression was also undetectable in porcine placental areolae.

In addition to specific regions of the trophoblast, staining was also observed in endometrial glands on D50 and D110 (Fig. 2c). However, staining in endometrial glands was inconsistent between different animals and locations, and was only weak, if any. Thus, the authenticity of this staining must be considered as questionable. However, the expression of aromatase in porcine endometrial glands would be consistent with earlier observations in which a considerable estrogen synthesis was also detected in endometrial samples during porcine pregnancy. Intriguingly, aromatase activities in the endometrium were two to three times as high as those in the placenta on D30, D60 and D90 (16 and Hopkins K. L.: In vitro characterization of aromatase, estrone sulfotransferase and estrone sulfatase activities in the porcine placenta and endometrium at 30, 60 and 90 days of gestation. Master thesis, Virginia Polytechnic Institute and State University, Blacksburg, Virginia, USA. http://vtechworks.lib.vt.edu/bitstream/ handle/10919/45793/LD5655.V855 1987.H664.pdf? sequence $=1 \&$ isAllowed $=y$ ). However, according to our immunohistochemical findings, overall aromatase 
expression in the endometrium, if any, is very low compared to that in the placenta. These divergences between observations still need to be explained, but they could possibly be due to problems with appropriate separation of fetal and maternal components when preparing tissue samples for the measurement of aromatase activity.

In summary, the results show that, unlike at the periimplantation stage, at which aromatase is apparently expressed in a nearly uniform manner throughout the trophoblast, in the developed porcine placenta, it is restricted to the specialized trophoblast lining the trough-shaped chorionic fossa regions. The formation of similar aromatase-positive structures at the flanks of chorionic folds adjacent to the free margins of outgrowing secondary and tertiary endometrial folds between mid and late gestation points to a role of placental estrogens in the formation of a more complex geometry of the feto-maternal interface as pregnancy progresses.

\section{References}

1. Abd-Elnaeim M. M., Derar I. R., Wilsher S., Allen W. R., Leiser R., Schuler G. Immunohistochemical localization of oestrogen receptors alpha and beta, progesterone receptor and aromatase in the equine placenta. Reprod. Domest. Anim. 2009, 44, 312-319.

2. Bazer F. W., Thatcher $W$. W.: Theory of maternal recognition of pregnancy in swine based on estrogen controlled endocrine versus exocrine secretion of prostaglandin F2alpha by the uterine endometrium. Prostaglandins 1977, 14, 397-400

3. Choi I., Collante W. R., Simmen R. C., Simmen F. A.: A developmental switch in expression from blastocyst to endometrial/placental-type cytochrome P450 aromatase genes in the pig and horse. Biol. Reprod. 1997, 56, 688-696.

4. Conley A. J., Christenson L. K., Ford S. P., Christenson R. K. Immunocytochemical localization of cytochromes P450 17 alpha-hydroxylase and aromatase in embryonic cell layers of elongating porcine blastocysts. Endocrinology 1994, 135, 2248-2254.

5. Conley A. J., Corbin C. J., Hughes A. L.: Adaptive evolution of mammalian aromatases: lessons from Suiformes. J. Exp. Zool. A Ecol. Genet. Physiol. 2009, 311, 346-357.

6. Conley A. J., Hinshelwood M.: Mammalian aromatases. Reproduction 2001, 121, 685-695.

7. Czarnowska E.: The ultrastructure of the trophoblast fossal regions in the pig placenta during pregnancy. Its steroidogenic features. Anat. Histol. Embryol. $1988,17,207-325$

8. Dantzer $V$ : Cell biological aspects of porcine placentation: a scanning and transmission electron microscopic study. Thesis (Ph. D.) Royal Veterinary and Agricultural University, Copenhagen 1986.

9. Ford S. P., Christenson R. K.: Blood flow to uteri of sows during the estrous cycle and early pregnancy: local effect of the conceptus on the uterine blood supply. Biol. Reprod. 1979, 21, 617-624

10. Friess A. E., Sinowatz F., Skolek-Winnisch R., Träutner W.: The placenta of the pig. I. Finestructural changes of the placental barrier during pregnancy. Anat. Embryol. (Berl.) 1980, 158, 179-191.

11. Friess A. E., Sinowatz F., Skolek-Winnisch R., Träutner W.: The placenta of the pig. II. The ultrastructure of the areolae. Anat. Embryol. (Berl.) 1981, 163, 43-53.

12. Geisert R. D., Meyer A. E., Pfeiffer C. A., Johns D. N., Lee K., Wells K. D., Spencer T. E., Prather R. S.: Gene editing to investigate role of conceptus factors in establishment of pregnancy in the pig. Reproduction 2021. REP-200604.R2. doi: 10.1530/REP-20-0604. Epub ahead of print. PMID: 33560267.

13. Goldstein M. H., Bazer F. W., Barron D. H.: Characterization of changes in volume, osmolarity and electrolyte composition of porcine fetal fluids during gestation. Biol. Reprod. 1980, 22, 1168-1180.

14. Knight J. W.: Aspects of placental estrogen synthesis in the pig. Exp. Clin Endocrinol. 1994, 102, 175-184.

15. Knight J. W., Bazer F. W., Thatcher W. W., Franke D. E., Wallace H. D. Conceptus development in intact and unilaterally hysterectomized-ovariectomized gilts: interrelations among hormonal status, placental development, fetal fluids and fetal growth. J. Anim. Sci. 1977, 44, 620-637.
16. Krzymowski T., Stefanczyk-Krzymowska S.: The oestrous cycle and early pregnancy - a new concept of local endocrine regulation. Vet. J. 2004, 168, 285-296.

17. Leiser R., Kaufmann P.: Placental structure: in a comparative aspect. Exp. Clin. Endocrinol. 1994, 102, 122-134.

18. Meyer A. E., Pfeiffer C. A., Brooks K. E., Spate L. D., Benne J. A., Cecil R., Samuel M. S., Murphy C. N., Behura S., McLean M. K., Ciernia L. A., Smith M. F., Whitworth K. M., Wells K. D., Spencer T. E., Prather R. S., Geisert $R$. D.: New perspective on conceptus estrogens in maternal recognition and pregnancy establishment in the pig. Biol. Reprod. 2019, 101, 148-161.

19. Ohtaki T., Moriyoshi M., Nakada K., Nakao T., Kawata K.: Radioimmunoassay of saliva estrone sulfate in pregnant sows. J. Vet. Med. Sci. 1997, 59, 759-763.

20. Robertson H. A., King G. J.: Plasma concentrations of progesterone, oestrone, oestradiol-17beta and of oestrone sulphate in the pig at implantation, during pregnancy and at parturition. J. Reprod. Fertil. 1974, 40, 133-141.

21. Robertson H. A., King G. J., Dyck G. W.: The appearance of oestrone sulphate in the peripheral plasma of the pig early in pregnancy. J. Reprod. Fertil. 1978, 52, 337-338.

22. Schuler G.: Steroid sulfates in domestic mammals and laboratory rodents. Dom. Anim. Endocrinol. 2021. Epub ahead of print, doi: 10.1016/ j.domaniend.2021.106622.

23. Schuler G., Fürbass R., Klisch K.: Placental contribution to the endocrinology of gestation and parturition. Anim. Reprod. 2018, 15 nSupplement 1, 822-842, doi: 10.21451/1984-3143-AR2018-0015

24. Schuler G., Greven H., Kowalewski M. P., Döring B., Özalp G. R., Hoffmann B. Placental steroids in cattle: hormones, placental growth factors or by-products of trophoblast giant cell differentiation? Exp. Clin. Endocrinol. Diabetes. 2008, 116, 429-436.

25. Turner K. J., Macpherson S., Millar M. R., McNeilly A. S., Williams K., Cranfield M., Groome N. P., Sharpe R. M., Fraser H. M., Saunders P. T: Development and validation of a new monoclonal antibody to mammalian aromatase. J. Endocrinol. 2002, 172, 21-30.

26. Ziecik A. J.: Old, new and the newest concepts of inhibition of luteolysis during early pregnancy in pig. Domest. Anim. Endocrinol. 2002, 23, 265-275.

27. Ziecik A. J., Waclawik A., Kaczmarek M. M., Blitek A., Jalali B. M., Andronowska A.: Mechanisms for the establishment of pregnancy in the pig. Reprod. Domest. Anim. 2011, 46 Suppl 3, 31-41.

Corresponding author: Prof. Gerhard Schuler, Clinic for Veterinary Obstetrics, Gynecology and Andrology of Large and Small Animals, Faculty of Veterinary Medicine, Justus-Liebig-University Giessen, Frankfurter Strasse 106, Giessen, Germany; e-mail: gerhard.schuler@vetmed.uni-giessen.de 